\title{
DROPLET BREAKUP IN THE LIQUID DROP MODEL WITH BACKGROUND POTENTIAL
}

\author{
STAN ALAMA, LIA BRONSARD, RUSTUM CHOKSI, AND IHSAN TOPALOGLU
}

\begin{abstract}
We consider a variant of Gamow's liquid drop model, with a general repulsive Riesz kernel and a long-range attractive background potential with weight $Z$. The addition of the background potential acts as a regularization for the liquid drop model in that it restores the existence of minimizers for arbitrary mass. We consider the regime of small $Z$ and characterize the structure of minimizers in the limit $Z \rightarrow 0$ by means of a sharp asymptotic expansion of the energy. In the process of studying this limit we characterize all minimizing sequences for the Gamow model in terms of "generalized minimizers".
\end{abstract}

\section{INTRODUCTION}

We consider the following variational problem:

$$
e_{Z}(M):=\inf \left\{\mathbf{E}_{Z}(\Omega): \Omega \subset \mathbb{R}^{d},|\Omega|=M\right\},
$$

where the energy functional $\mathbf{E}_{Z}$ is defined as

$$
\mathbf{E}_{Z}(\Omega):=\operatorname{Per}(\Omega)+\int_{\Omega} \int_{\Omega} \frac{d x d y}{|x-y|^{s}}-Z \int_{\Omega} \frac{d x}{|x|^{p}}
$$

with $0<p<s<d$ and $d \geqslant 2$. Here the first term is the perimeter of the set $\Omega$ in the sense of Caccioppoli and is given by

$$
\operatorname{Per}(\Omega)=\sup \left\{\int_{\Omega} \operatorname{div} \phi d x: \phi \in C_{0}^{1}\left(\mathbb{R}^{d} ; \mathbb{R}^{d}\right),\|\phi\|_{L^{\infty}\left(\mathbb{R}^{d}\right)} \leqslant 1\right\} .
$$

Our main motivation for this problem and the consideration of the small $Z$ regime stems from Gamow's liquid drop model [17] which successfully models the shape of an atomic nucleus. Gamow's model is essentially equivalent to the minimization problem (1) with $d=3$, Coulombic repulsion $s=1$, and $Z=0$ :

$$
\text { minimize } \operatorname{Per}(\Omega)+\int_{\Omega} \int_{\Omega} \frac{d x d y}{|x-y|} \quad \text { over } \quad \Omega \subset \mathbb{R}^{3} \quad \text { with } \quad|\Omega|=M .
$$

Date: October 11, 2018.

1991 Mathematics Subject Classification. 35Q40, 35Q70, 49Q20, 49S05, 82D10.

Key words and phrases. liquid drop model, droplet breakup, background potential, nonlocal isoperimetric problem, generalized minimizer, concentration-compactness method. 
This problem recently resurfaced in the context of the Ohta-Kawasaki model for self-assembly of diblock copolymers (cf. [9, 10]), and has since attracted much mathematical interest (cf. [6, 14, 15, 18, 20, 25, 29, 30, as well as [8] for a general overview). One of the fundamental characteristics of the liquid drop model is that it predicts the spherical shape of small nuclei and the non-existence of arbitrarily large nuclei. It is precisely the competition between opposing forces (the surface tension and Coulombic repulsion) which makes proving these predictions non-trivial. The non-existence of minimizers for large $M$ is associated with the breakup of droplets tending to infinity.

From a physical point of view, though, one might expect other forces to be present which restore existence for larger values of $M$, predicting a structured configuration of droplets. One way to introduce such effects is to introduce an attractive "background nucleus", which is effected by adding to (3) an external attractive potential of the form

$$
V(x)=-\frac{Z}{|x|^{p}}
$$

for $Z>0$ and $0<p \leqslant 1$. Here we take the "background nucleus" to be centered at the origin, and of longer range, in the sense that they have slower decay than the Coulombic nonlocal interaction term. The physical case of $p=1$ (Coulombic attraction) was recently considered by Lu and Otto [24], and by Frank, Nam and van den Bosch [16] where it was proved that the effect of $V$ simply increases the critical threshold in $M$ for the non-existence of minimizers. On the other hand, choosing a potential with $p<1$ restores existence for all $M$ (cf. Theorem 1 and [3]); we may think of the addition of the attractive long-range potential as regularizing the generalized liquid drop model (2). We then focus on the structure of minimizers in the small $Z$ regime. In doing so, we completely describe particular configurations of generalized minimizers (cf. [20], Definition 3) of the liquid drop model.

Our first result confirms that the presence of the external potential (44) with $p<s$ indeed restores existence for all masses $M>0$.

Theorem 1. For all $Z>0$ and for any $M>0$, the minimum $e_{Z}(M)$ is attained.

This result is a generalization of the result in [3], and for convenience we will present an outline of the proof (which differs from that in [3]) in section 2. Our principal interest is in studying minimizers of $\mathbf{E}_{Z}$ in the limit $Z \rightarrow 0$. For $d \geqslant 2$, it is well-known that there exists $m^{*}=m^{*}(d, s)>0$ such that the $Z=0$ problem,

$$
e_{0}(M):=\inf \left\{\mathbf{E}_{0}(\Omega): \Omega \subset \mathbb{R}^{d},|\Omega|=M\right\}
$$


DROPLET BREAKUP IN THE LIQUID DROP MODEL WITH BACKGROUND POTENTIAL 3 does not admit minimizers for $M>m^{*}$ and $s \in(0,2)$ (see [18, Theorem 2.5] and [19, Theorem 3.3], and also [25] and [14] for the case $d=3, s=1$ ). Thus, when $M>m^{*}$ a sequence of minimizers $\Omega_{Z}$ of the functional $\mathbf{E}_{Z}$ must lose compactness as $Z \rightarrow 0$. We show this is indeed the case: for small $Z>0, \Omega_{Z}$ is composed of a finite number of widely spaced disjoint compact components, separated by a distance on the order of $Z^{-1 /(s-p)}$. Moreover, we show that the components are arranged in a way which (after rescaling by $Z^{1 /(s-p)}$ ) optimizes a discrete interaction energy,

$$
\mathbf{F}_{N, \underline{m}}\left(y_{0}, \ldots, y_{N}\right):=\sum_{\substack{i, j=0 \\ i \neq j}}^{N} \frac{m^{i} m^{j}}{\left|y_{i}-y_{j}\right|^{s}}-\sum_{i=1}^{N} \frac{m^{i}}{\left|y_{i}\right|^{p}},
$$

where $\underline{m}=\left(m^{0}, \ldots, m^{N}\right)$ with $\sum_{i=0}^{N} m^{i}=M$, and $y_{0}, \ldots, y_{N}$ in the admissible class

$$
\Sigma_{N}:=\left\{\left(y_{0}, \ldots, y_{N}\right) \subset \mathbb{R}^{3(d+1)}: y_{0}=0\right\} .
$$

Our main result describes the structure of minimizers of $\mathbf{E}_{Z}$ for small $Z>0$ :

Theorem 2. Let $\Omega_{Z}$ be minimizers of $\mathbf{E}_{Z}$ for $Z>0$. Then for any sequence $Z \rightarrow 0$ there exists a subsequence $Z_{n} \rightarrow 0$ so that either

(A) there exists a set $E^{0}$ with $\left|E^{0}\right|=M$ which minimizes $e_{0}(M)$, for which $\Omega_{Z_{n}} \rightarrow$ $E^{0}$ globally, i.e., $\chi_{\Omega_{Z_{n}}} \rightarrow \chi_{E^{0}}$ in $L^{1}\left(\mathbb{R}^{d}\right)$ as $n \rightarrow \infty$; or

(B) there exist:

(i) $N \in \mathbb{N}$;

(ii) $\left(m^{0}, \ldots, m^{N}\right), m^{i}>0$ with $\sum_{i=0}^{N} m^{i}=M$;

(iii) $x_{n}^{0}, \ldots, x_{n}^{N} \in \mathbb{R}^{d}$, with $x_{n}^{0}=0$, and $\left|x_{n}^{i}\right| \rightarrow \infty$ for $i \neq 0$. and $\left|x_{n}^{i}-x_{n}^{j}\right| \rightarrow \infty$ for $i \neq j$ as $n \rightarrow \infty$;

(iv) $E^{0}, \ldots, E^{N}$ compact sets of finite perimeter, with $\left|E^{i}\right|=m^{i} \neq 0$ for $i=$ $0, \ldots, N$

such that $\Omega_{n}:=\Omega_{Z_{n}}$ satisfies the following:

$$
\begin{gathered}
\partial^{*} \Omega_{n} \in C^{1, \frac{1}{2}}, \quad \text { and for fixed } R>0 \text { such that all } E^{i} \subset B_{R}(0), \\
\left(\partial^{*} \Omega_{n}-x_{n}^{i}\right) \cap B_{R}(0) \rightarrow \partial^{*} E^{i} \text { in } C^{1, \alpha} \text { for all } \alpha \in(0,1 / 2) ; \\
\qquad \Omega_{n} \triangle\left[E^{0} \cup \bigcup_{i=1}^{N}\left(E^{i}+x_{n}^{i}\right)\right] \mid \longrightarrow 0 ;
\end{gathered}
$$

$E^{i}$ attains the minimum in (5), i.e., $e_{0}\left(m^{i}\right)=\mathbf{E}_{0}\left(E^{i}\right), i=0,1, \ldots, N$;

$$
\left\{\begin{array}{c}
Z_{n}^{\frac{1}{s-p}} x_{n}^{i} \longrightarrow y_{i} \text { as } n \rightarrow \infty, i=1, \ldots, N, \\
\text { where }\left(0, y_{1}, \ldots, y_{N}\right) \text { minimize } \mathbf{F}_{N, \underline{m}} \text { over } \Sigma_{N} .
\end{array}\right.
$$


Here $\partial^{*}$ denotes the reduced boundary of a set. By regularity theory of perimeter minimizing sets (and more generally, $(\omega, r)$-minimizers of perimeters) the topological boundary $\partial E^{i}$ differs from the reduced boundary by a set of small Hausdorff dimension, $\operatorname{dim}_{\mathcal{H}}\left(\partial E^{i} \backslash \partial^{*} E^{i}\right) \leqslant n-8$.

We note the distinction between the existence result in Theorem 2 of $\mathbf{E}_{Z}$ and those of the Gamow functional: for Gamow's model, minimizers only exist for small mass $M$, and must be connected. On the other hand, for $Z>0$ but small, minimizers of $\mathbf{E}_{Z}$ always exist for any $M$ but must be disconnected for mass $M>m^{*}$.

The proof of Theorem 2 relies on a general concentration-compactness lemma (Lemma 6) for minimizing sequences of $\mathbf{E}_{Z}$. We prove this result using a recent compactness result for sequences of Caccioppoli sets by Frank and Lieb [15]. It is in this lemma that we first encounter the effect of splitting of the support of minimizers, when the total mass is large. The resulting structure (as described by conclusions (9) and (10) of Theorem 2, ) was formalized by Knüpfer, Muratov, and Novaga [20, Definition 4.3]); we adapt their definition to $\mathbf{E}_{Z}$ :

Definition 3. Let $Z \geqslant 0$ and $M>0$. A generalized minimizer of $\mathbf{E}_{Z}$ is a finite collection $\left(E^{0}, E^{1}, \ldots, E^{N}\right)$ of sets of finite perimeter, such that:

(i) $\left|E^{i}\right|:=m^{i}, i=0,1, \ldots, N$, with $\sum_{i=0}^{N} m^{i}=M$;

(ii) $E^{0}$ attains the minimum in $e_{Z}\left(m^{0}\right)$ and $E^{i}$ attains $e_{0}\left(m^{i}\right), i=1, \ldots, N$;

(iii) $e_{Z}(M)=e_{Z}\left(m^{0}\right)+\sum_{i=1}^{N} e_{0}\left(m^{i}\right)$.

In [20] the authors prove the existence of generalized minimizers for the Gamow problem $Z=0$. Here we improve their result: it follows immediately from the concentration lemma (Lemma 6) that any minimizing sequence of $\mathbf{E}_{Z}$, for $Z \geqslant 0$, is completely characterized (up to sets of vanishingly small measure, and along subsequences) by a generalized minimizer:

Corollary 4. Let $Z \geqslant 0, M>0$, and suppose $\left\{\Omega_{n}\right\}_{n \in \mathbb{N}}$ is any minimizing sequence for $e_{Z}(M)$. Then, there is a subsequence, $N \geqslant 0$, and a generalized minimizer $\left(E^{0}, E^{1}, \ldots, E^{N}\right)$ of $\mathbf{E}_{Z}$, with

$$
\left|\Omega_{n} \triangle\left[E^{0} \cup \bigcup_{i=1}^{N}\left(E^{i}+x_{n}^{i}\right)\right]\right| \longrightarrow 0,
$$

for a sequence of translations $\left(x_{n}^{i}\right)_{n \in \mathbb{N}}^{i=1, \ldots, N}$.

In the context of generalized minimizers, Theorem 2 asserts that the family $\Omega_{Z}$ of minimizers of $\mathbf{E}_{Z}$ makes a particular selection of a generalized minimizer (the sets $\left\{E^{i}\right\}_{i=1, \ldots, N}$ obtained in the theorem,) for the generalized liquid drop problem $\mathbf{E}_{0}$. We note that the special choice of generalized minimizer obtained this way may not 
be canonical, in the sense of viscosity solutions in PDE; the sets and the pattern they form as $Z \rightarrow 0$ depend on the choice of external potential.

The concept of generalized minimizers is a familiar one in applications of concentration compactness, and is intimately related to the notion of "critical points at infinity", introduced by Bahri [4] in his study of existence of solutions for Yamabetype equations and other PDE problems with loss of compactness. (See [31] for other contexts involving critical points or functionals "at infinity".)

In addition to the concentration-compactness structure given in Lemma 6, the proof of Theorem 2 requires an expansion of the energy $\mathbf{E}_{Z}$ up to the third-order term in $Z$ (see Remark 12 below). In order to establish this, we combine the compactness of a sequence of minimizers $\Omega_{Z}$ with regularity results stemming from the classical regularity properties of the perimeter functional improving the error estimates in [15]. Similar methods were employed in a previous paper [2], concerning concentration of droplets in a sharp interface model of diblock copolymers under confinement.

We note that the limiting finite dimensional energy $\mathbf{F}_{N, \underline{m}}\left(y_{0}, \ldots, y_{N}\right)$ (unlike its counterpart in [2]) is not coercive, and so it is not clear a priori that minimizing sequences for this energy should not split, with some number of points diverging to infinity. However, in Proposition 8 we will show that this finite dimensional discrete variational problem attains its minimizer for all choices of $N$ and the masses $\underline{m}$, a result which we will use in studying the limit $Z \rightarrow 0$ but which is itself of independent interest.

In light of Theorem 2, it is natural to ask if the family of functionals $\mathbf{E}_{Z}$ has a second-order $\Gamma$-limit, involving generalized minimizers of the Gamow functional and the finite dimensional interaction energy $\mathbf{F}_{N, \underline{m}}$. Such a result would imply the existence of local minimizers for $\mathbf{E}_{Z}$, with small $Z>0$. However, the method to prove Theorem 2 uses regularity properties of minimizers in a fundamental way, and does not directly extend to the more general setting of $\Gamma$-convergence.

Finally, Bonacini and Cristoferi [6, Theorem 2.11] have shown that there exists a critical value $\bar{s}(d)$ of the power in the Riesz kernel such that if $s \in(0, \bar{s}(d))$, then the minimizers of $e_{0}(M)$ (when they exist) must be balls. In other words, for small $s$, the critical mass for existence exactly coincides with the critical value at which minimizers must be balls. In this case, we have a near-complete description of minimizers for small $Z>0$, as a finite configuration of balls of equal radius:

Theorem 5. Assume $0<s<\bar{s}(d)$, and $0<p<s<d$. Then, the sets $E^{i}$ appearing in Theorem 2 are all balls with equal volume $m^{i}=M /(N+1), i=0,1, \ldots, N$. 
The idea behind the proof of Theorem 5 is that each diverging component of a minimizer of $\mathbf{E}_{Z}$ inherits the same Lagrange multiplier, and so each element $E^{i}$ of the generalized minimizer "at infinity" satisfies the same Euler-Lagrange equation. When the minimizers are balls, the radius is uniquely determined by the Lagrange multiplier. As the first part of the argument holds for any values of $s, d, M$, we in fact conjecture that the equipartition of mass between the components of the generalized minimizers is true whether the minimizers are balls or not.

The liquid drop model (3) was introduced to describe nuclear structure. In fact it appears in various other contexts (mathematical and physical) to describe systems with competition between short- and long-range effects on many scales, from the nuclear to nanoscale (in condensed matter systems), to centimeter scale (for fluids and autocatalytic reaction-diffusion systems, ) and even on cosmological scales. In the original quantum context for the atomic nucleus, we do not know of any physical interpretation of such a background potential, even one of Coulombic type $(p=1)$. However, in the wider context (particularly the cosmological context), consideration of super-Newtonian forces appears in several theories. In fact, the validity of Newton's law at long distances has been a longstanding interest in physics. As Finzi notes in [13] stability of clusters of galaxies implies stronger attractive forces at long distances than that predicted by Newton's law. Motivated by similar observations, in [27] Milgrom introduced the modified Newtonian dynamics (MOND) theory which suggests that the gravitational force experienced by a star in the outer regions of a galaxy must be stronger than Newton's law (cf. [5, [7, 28]).

\section{Concentration-Compactness and existence}

In this section we prove the basic concentration-compactness structure of minimizing sequences for $\mathbf{E}_{Z}$. While this result could be adapted, for example, from the classical theory of Lions (see [23] or Lemma 1 in the Appendix of [22]), or from compactness results for minimizing clusters as in [26, Chapter 29], here we use a recent compactness result by Frank and Lieb [15] which is particularly well-suited for our purposes.

We will say that a sequence of sets $E_{n} \rightarrow E$ globally in $\mathbb{R}^{d}$ if the measure of the symmetric difference $\left|E_{n} \triangle E\right| \rightarrow 0$. We similarly say that $E_{n} \rightarrow E$ locally if for every compact $K \subset \mathbb{R}^{d},\left(K \cap E_{n}\right) \rightarrow(K \cap E)$ globally. Global convergence is thus equivalent to convergence of the characteristic functions $\chi_{E_{n}} \rightarrow \chi_{E}$ in $L^{1}\left(\mathbb{R}^{d}\right)$, while local convergence is merely $L_{\text {loc }}^{1}$ convergence of the characteristic functions.

Lemma 6. Let $Z \in[0, \infty)$ be fixed, and $\left\{\Omega_{n}\right\}_{n \in \mathbb{N}}$ a minimizing sequence for $e_{Z}(M)$. Then there exists a subsequence such that either 
DROPLET BREAKUP IN THE LIQUID DROP MODEL WITH BACKGROUND POTENTIAL 7

(A) there exists a set $E^{0}$ with $\left|E^{0}\right|=M$ which minimizes $e_{Z}(M)$, for which $\Omega_{n} \rightarrow E^{0}$ globally, i.e., $\chi_{\Omega_{n}} \rightarrow \chi_{E^{0}}$ in $L^{1}\left(\mathbb{R}^{d}\right)$ as $n \rightarrow \infty$; or

(B) there exist $N \in \mathbb{N} ;\left\{x_{n}^{1}, \ldots, x_{n}^{N}\right\}_{n \in \mathbb{N}} \subset \mathbb{R}^{d}$, with $\left|x_{n}^{i}\right| \rightarrow \infty$ and sets of finite perimeter $\left\{F_{n}^{0}, \ldots, F_{n}^{N}, \Omega_{n}^{N}\right\}_{n \in \mathbb{N}}$ such that $\left|x_{n}^{i}-x_{n}^{j}\right| \rightarrow \infty, i \neq j ;$ with

$$
\Omega_{n}=F_{n}^{0} \cup\left[\bigcup_{i=1}^{N}\left(F_{n}^{i}+x_{n}^{i}\right)\right] \cup \Omega_{n}^{N},
$$

a disjoint union with components satisfying the following:

(i) $\Omega_{n}^{N} \rightarrow \emptyset$ and $F_{n}^{i} \rightarrow E^{i}$, globally in $\mathbb{R}^{d}$, with $m^{i}:=\left|E^{i}\right|>0$ for all $i=$ $1, \ldots, N$ and $\left|E^{0}\right|>0$ for $Z>0$;

(ii) $M=\sum_{i=0}^{N}\left|E^{i}\right|=\lim _{n \rightarrow \infty}\left(\sum_{i=0}^{N}\left|F_{n}^{i}\right|+\left|\Omega_{n}^{N}\right|\right)$;

(iii) $E^{i}$ attain the minimimum for $e_{0}\left(m^{i}\right)$ for each $i=1, \ldots, N$;

(iv) $E^{0}$ attains the minimum for $e_{Z}\left(m^{0}\right)$;

(v) $e_{Z}(M) \geqslant e_{Z}\left(m^{0}\right)+\sum_{i=1}^{N} e_{0}\left(m^{i}\right)$.

As mentioned in the introduction (see [20, Definition 4.3],) the collection of sets $\left\{E^{0}, \ldots, E^{N}\right\}_{n \in \mathbb{N}}$ are referred to as a generalized minimizer of $\mathbf{E}_{Z}$ for any $Z \geqslant 0$. Knüpfer, Muratov, and Novaga prove the existence of generalized minimizers for the case $Z=0$ by considering a truncation of the energy $\mathbf{E}_{0}$ and by obtaining density bounds for minimizers of the truncated energy (cf. [20, Theorem 4.5]). Our approach in proving Lemma 6 is more direct, and provides qualitative information about the structure of minimizing sequences that we exploit in Theorem 2. In particular, Corollary 4 follows, since $F_{n}^{i} \rightarrow E^{i}$ and (12) then imply

$$
\lim _{n \rightarrow \infty}\left|\Omega_{n} \triangle\left(E^{0} \cup \bigcup_{i=1}^{N}\left(E^{i}+x_{n}^{i}\right)\right)\right|=0 .
$$

Before going back to Lemma 6, we need the following result to conveniently deal with the confinement term.

Lemma 7. Assume $A_{n}$ is a sequence of measurable sets with $\left|A_{n}\right|=M$ and $A_{n} \rightarrow 0$ locally (that is, $\left|A_{n} \cap K\right| \rightarrow 0$ for any compact $K$.) Then,

$$
\lim _{n \rightarrow \infty} \int_{A_{n}} \frac{1}{|x|^{p}} d x=0
$$

The proof is an elementary exercise in real analysis, obtained by truncating $|x|^{-p}$ both vertically and laterally. 
We also require the following subadditivity condition, which follows from the same arguments as Lemma 4 of [24]: for any values $0<m^{\prime}<m$, and any $Z \geqslant 0$,

$$
e_{Z}(m) \leqslant e_{Z}\left(m^{\prime}\right)+e_{0}\left(m-m^{\prime}\right)
$$

Proof of Lemma 6. Let $Z \geqslant 0$ be fixed and $\Omega_{n}$ a minimizing sequence for $e_{Z}(M)$. We prove this lemma in several step.

Step 1: Passing to the limit directly. By the compact embedding of $B V\left(\mathbb{R}^{d}\right)$ in $L_{\text {loc }}^{1}\left(\mathbb{R}^{d}\right)$ (see e.g. [26, Corollary 12.27]) there exists a subsequence and a set of finite perimeter $E^{0} \subset \mathbb{R}^{d}$ so that $\Omega_{n} \rightarrow E^{0}$ locally, that is, $\chi_{\Omega_{n}} \rightarrow \chi_{E^{0}}$ in $L_{\mathrm{loc}}^{1}\left(\mathbb{R}^{d}\right)$. (At this point, we admit the possibility that $\left|E^{0}\right|=0$, but in fact we will see in Step 3 that $\left|E^{0}\right|>0$.)

We claim that if the limit set $\left|E^{0}\right|=M$, then case (A) holds and we are done. Indeed, since $\left\{\Omega_{n}\right\}_{n \in \mathbb{N}}$ is locally convergent, a subsequence converges almost everywhere in $\mathbb{R}^{d}$. In addition, the measures of the sets converge, that is, $\left|\Omega_{n}\right|=M=\left|E^{0}\right|$, so by the Brezis-Lieb Lemma [21, Theorem 1.9] we may then conclude that (along a subsequence) $\Omega_{n} \rightarrow E^{0}$ globally. By the lower semicontinuity of the perimeter (see [26, Proposition 4.29]) we have

$$
\text { Per } E^{0} \leqslant \liminf _{n \rightarrow \infty} \operatorname{Per} \Omega_{n}
$$

On the other hand, [15, Lemma 2.3] implies that the nonlocal part is lower semicontinuous, as well, that is,

$$
\mathcal{D}\left(E^{0}, E^{0}\right) \leqslant \liminf _{n \rightarrow \infty} \mathcal{D}\left(\Omega_{n}, \Omega_{n}\right) \quad \text { where } \quad \mathcal{D}(E, F):=\int_{E} \int_{F} \frac{d x d y}{|x-y|^{s}} .
$$

To pass to the limit in the confinement term, we apply Lemma 7 to the sequence $\left(\Omega_{n} \backslash E^{0}\right) \rightarrow \emptyset$ locally, and together with the above we have

$$
\mathbf{E}_{Z}\left(E^{0}\right) \leqslant \liminf _{n \rightarrow \infty} \mathbf{E}_{Z}\left(\Omega_{n}\right)
$$

Therefore we conclude that $E^{0}$ attains the minimum value of $\mathbf{E}_{Z}$, and the proof is complete for case (A).

In the following we may thus assume that $m^{0}:=\left|E^{0}\right|<M$.

Step 2: Concentration-compactness. In case $m^{0}=\left|E^{0}\right|<M$, by [15, Lemma 2.2] (with no translation necessary, i.e., $x_{n}^{0}=0$ ) there exist radii $r_{n}^{0} \in(0, \infty)$ such that for

$$
F_{n}^{0}=\Omega_{n} \cap B_{r_{n}^{0}}(0) \quad G_{n}^{0}=\Omega_{n} \backslash \overline{B_{r_{n}^{0}}}(0)
$$


DROPLET BREAKUP IN THE LIQUID DROP MODEL WITH BACKGROUND POTENTIAL 9

where $F_{n}^{0} \rightarrow E^{0}$ globally, $G_{n}^{0} \rightarrow \emptyset$ locally as $n \rightarrow \infty$ with $m_{n}^{0}:=\left|F_{n}^{0}\right| \rightarrow\left|E^{0}\right|=$ $m^{0}<M$, and

$$
\lim _{n \rightarrow \infty}\left(\operatorname{Per}\left(\Omega_{n}\right)-\operatorname{Per}\left(F_{n}^{0}\right)-\operatorname{Per}\left(G_{n}^{0}\right)\right)=0, \quad \liminf _{n \rightarrow \infty} \operatorname{Per}\left(F_{n}^{0}\right) \geqslant \operatorname{Per}\left(E^{0}\right) .
$$

In addition, again by [15, Lemma 2.3],

$$
\mathcal{D}\left(\Omega_{n}, \Omega_{n}\right)=\mathcal{D}\left(F_{n}^{0}, F_{n}^{0}\right)+\mathcal{D}\left(G_{n}^{0}, G_{n}^{0}\right)+o(1)=\mathcal{D}\left(E^{0}, E^{0}\right)+\mathcal{D}\left(G_{n}^{0}, G_{n}^{0}\right)+o(1) .
$$

Finally, by Lemma 7 , the confinement term is absent for $G_{n}^{0}$, which tends to zero locally. In conclusion, we have a splitting of the energy,

$$
\mathbf{E}_{Z}\left(\Omega_{n}\right)=\mathbf{E}_{Z}\left(E^{0}\right)+\mathbf{E}_{0}\left(G_{n}^{0}\right)+o(1) .
$$

We define $\Omega_{n}^{0}:=G_{n}^{0}$, with $\left|\Omega_{n}^{0}\right|=M-m_{n}^{0}=M-m^{0}+o(1)>0$, and begin an iterative process of locating escaping concentrations of mass, as in the concentrationcompactness lemma of Lions (cf. [23]). By [15, Proposition 2.1], there is a set $E^{1}$ of positive measure and a sequence of points $x_{n}^{1} \in \mathbb{R}^{d}$ for which $\Omega_{n}^{0}-x_{n}^{1} \rightarrow E^{1}$ locally. Since $\Omega_{n}^{0} \rightarrow 0$ locally, it follows that $\left|x_{n}^{1}\right| \rightarrow \infty$. In addition, $\left|E^{1}\right| \in\left(0, M-m^{0}\right]$ and $\operatorname{Per}\left(E^{1}\right) \leqslant \liminf _{n \rightarrow \infty} \operatorname{Per}\left(\Omega_{n}^{0}\right)$. In case of nonuniqueness of such translates, we define

$$
\begin{aligned}
\mu\left(\left\{\Omega_{n}^{0}\right\}\right):=\sup \left\{|A|: \text { there exist } A \subset \mathbb{R}^{d}\right. \text { and } \\
\left.\left.\qquad \xi_{n}\right\} \subset \mathbb{R}^{d} \text { such that } \Omega_{n}^{0}-\xi_{n} \rightarrow A \text { locally }\right\} .
\end{aligned}
$$

We may thus choose $\left\{x_{n}^{1}\right\}$ and $E^{1}$ such that $\left|E^{1}\right|>\frac{1}{2} \mu\left(\left\{\Omega_{n}^{0}\right\}\right)$.

Applying [15, Lemma 2.2] as above, there exist radii $r_{n}^{1} \in(0, \infty)$ such that if we define

$$
F_{n}^{1}=\left(\Omega_{n}^{0}-x_{n}^{1}\right) \cap B_{r_{n}^{1}}(0) \quad G_{n}^{1}=\left(\Omega_{n}^{0}-x_{n}^{1}\right) \backslash \overline{B_{r_{n}^{1}}}(0)
$$

then $F_{n}^{1} \rightarrow E^{1}$ globally, $G_{n}^{1} \rightarrow \emptyset$ locally as $n \rightarrow \infty$, with $m_{n}^{1}:=\left|F_{n}^{1}\right| \rightarrow\left|E^{1}\right|=$ : $m^{1} \in\left(0, M-m^{0}\right]$,

$$
\begin{aligned}
0=\lim _{n \rightarrow \infty}\left(\operatorname{Per}\left(\Omega_{n}^{0}\right)-\operatorname{Per}\right. & \left.\left(F_{n}^{1}\right)-\operatorname{Per}\left(G_{n}^{1}\right)\right) \\
& =\lim _{n \rightarrow \infty}\left(\operatorname{Per}\left(\Omega_{n}\right)-\operatorname{Per}\left(F_{n}^{0}\right)-\operatorname{Per}\left(F_{n}^{1}\right)-\operatorname{Per}\left(G_{n}^{1}\right)\right)
\end{aligned}
$$

and $\liminf \operatorname{in}_{n \rightarrow \infty} \operatorname{Per}\left(F_{n}^{1}\right) \geqslant \operatorname{Per}\left(E^{1}\right)$. Finally, by [15, Lemma 2.3],

$$
\begin{aligned}
\mathcal{D}\left(\Omega_{n}, \Omega_{n}\right) & =\mathcal{D}\left(F_{n}^{0}, F_{n}^{0}\right)+\mathcal{D}\left(\Omega_{n}^{0}, \Omega_{n}^{0}\right) \\
& =\mathcal{D}\left(F_{n}^{0}, F_{n}^{0}\right)+\mathcal{D}\left(F_{n}^{1}, F_{n}^{1}\right)+\mathcal{D}\left(G_{n}^{1}, G_{n}^{1}\right)+o(1) \\
& =\mathcal{D}\left(E^{0}, E^{0}\right)+\mathcal{D}\left(E^{1}, E^{1}\right)+\mathcal{D}\left(G_{n}^{1}, G_{n}^{1}\right)+o(1)
\end{aligned}
$$

In particular,

$$
\mathbf{E}_{Z}\left(\Omega_{n}\right) \geqslant \mathbf{E}_{Z}\left(E^{0}\right)+\mathbf{E}_{0}\left(E^{1}\right)+\mathbf{E}_{0}\left(G_{n}^{1}\right)+o(1) .
$$


If $\left|G_{n}^{1}\right| \rightarrow 0$, the process terminates with $N=1$. If not, we let $\Omega_{n}^{1}:=G_{n}^{1}+x_{n}^{1}$, and repeat the above procedure with $\mu\left(\left\{\Omega_{n}^{1}\right\}\right) \in\left(0, M-m^{0}-m^{1}\right]$, iteratively generating an at most countable collection of concentration sets $F_{n}^{i} \rightarrow E^{i}$ and remainder sets $\Omega_{n}^{i}, i=1,2, \ldots$, satisfying

$$
\begin{gathered}
\Omega_{n}^{i-1}=\left[F_{n}^{i}+x_{n}^{i}\right] \cup \Omega_{n}^{i}, \quad \text { a disjoint union, } \\
\Omega_{n}=\Omega_{n}^{k} \cup\left[\bigcup_{i=0}^{k}\left(F_{n}^{i}+x_{n}^{i}\right)\right], \quad\left|x_{n}^{i}-x_{n}^{j}\right| \rightarrow \infty, i \neq j, \\
M=\sum_{i=0}^{k} m_{n}^{i}+\lim _{n \rightarrow \infty}\left|\Omega_{n}^{k}\right|=\sum_{i=0}^{k} m^{i}+\lim _{n \rightarrow \infty}\left|G_{n}^{k}\right|, \\
m^{k} \geqslant \frac{1}{2} \mu\left(\left\{\Omega_{n}^{k-1}\right\}\right), \\
\mathbf{E}_{Z}\left(\Omega_{n}\right) \geqslant \mathbf{E}_{Z}\left(\Omega_{n}^{k}\right)+\mathbf{E}_{Z}\left(E^{0}\right)+\sum_{i=1}^{k} \mathbf{E}_{0}\left(E^{i}\right)+o(1)
\end{gathered}
$$

for $k \in \mathbb{N}$. We note that the decomposition in (21) is disjoint, with $\Omega_{n}^{k} \rightarrow \emptyset$ locally.

In case $\left|\Omega_{n}^{N}\right| \rightarrow 0$ for some finite $N \in \mathbb{N}$, the process terminates and the decomposition is finite. If the number of components $E^{i}$ is countable, then by (22) we must have $m^{i} \rightarrow 0$ as $i \rightarrow \infty$, and hence $\mu\left(\left\{\Omega_{n}^{k}\right\}\right) \rightarrow 0$ as $k \rightarrow \infty$, by (23). We may then conclude that the iteration exhausts all of the mass, and

$$
M=\sum_{i=0}^{\infty} m^{i}
$$

Step 3: If $Z>0$, then $\left|E^{0}\right| \neq 0$. Suppose the contrary, i.e., that $\left|E^{0}\right|=0$. Define $\tilde{\Omega}_{n}:=\Omega_{n}-x_{n}^{1}$, and so by the above construction $\tilde{\Omega}_{n} \rightarrow E^{1}$ and $\tilde{\Omega}_{n} \backslash F_{n}^{1}=G_{n}^{1} \rightarrow \emptyset$ locally. Thus, by Lemma 7, for any $i \neq 1$,

$$
\lim _{n \rightarrow \infty} \int_{\tilde{\Omega}_{n} \backslash F_{n}^{1}} \frac{1}{|x|^{p}} d x=0 .
$$

Since the perimeter and nonlocal terms in $\mathbf{E}_{Z}$ are translation invariant, we arrive at

$$
\mathbf{E}_{Z}\left(\tilde{\Omega}_{n}\right)-\mathbf{E}_{Z}\left(\Omega_{n}\right)=-Z \int_{F_{n}^{1}} \frac{1}{|x|^{p}} d x+o(1)=-Z \int_{E^{1}} \frac{1}{|x|^{p}} d x+o(1)<0,
$$

a contradiction.

Step 4: The sets $E^{i}$ are minimal, and there are finitely many.

By Lemma 7 ,

$$
\liminf _{n \rightarrow \infty} \mathbf{E}_{Z}\left(\Omega_{n}^{k}\right)=\liminf _{n \rightarrow \infty}\left[\mathbf{E}_{0}\left(\Omega_{n}^{k}\right)-Z \int_{\Omega_{n}^{k}}|x|^{-p} d x\right]=\liminf _{n \rightarrow \infty} \mathbf{E}_{0}\left(\Omega_{n}^{k}\right) \geqslant 0 .
$$


DROPLET BREAKUP IN THE LIQUID DROP MODEL WITH BACKGROUND POTENTIAL11

Thus, as (24) holds for all $k \in \mathbb{N}$, we have: we then have

$$
\mathbf{E}_{Z}\left(\Omega_{n}\right) \geqslant \mathbf{E}_{Z}\left(E^{0}\right)+\sum_{i=1}^{\infty} \mathbf{E}_{0}\left(E^{i}\right)-o(1) .
$$

We may then conclude,

$$
\begin{aligned}
e_{Z}(M)+o(1) \geqslant \mathbf{E}_{Z}\left(\Omega_{n}\right) & \geqslant \mathbf{E}_{Z}\left(E^{0}\right)+\sum_{i=1}^{\infty} \mathbf{E}_{0}\left(E^{i}\right)-o(1) \\
& \geqslant e_{Z}\left(m^{0}\right)+\sum_{i=1}^{\infty} e_{0}\left(m^{i}\right)-o(1) \\
& \geqslant e_{Z}(M)-o(1)
\end{aligned}
$$

by the subadditivity condition (13) of $e_{Z}$. Matching the upper and lower bounds we have,

$$
\left(\mathbf{E}_{Z}\left(E^{0}\right)-e_{Z}\left(m^{0}\right)\right)+\sum_{i=1}^{\infty}\left[\mathbf{E}_{0}\left(E^{i}\right)-e_{0}\left(m^{i}\right)\right] \leqslant 0 .
$$

Since each term is nonnegative, each must be zero, and so each set $E^{i}, i=0,1, \ldots$ is minimal.

Lastly, as the series converges we must have $e_{0}\left(m^{i}\right) \rightarrow 0$ as $i \rightarrow \infty$, and from this fact we may conclude that only finitely many of $m^{i}$ are nonzero. This follows almost verbatim as in [9, Lemma 4.4], so we sketch the main idea here for completeness. Now let $m^{* *}>0$ be the constant such that $e_{0}$ is attained uniquely by a ball of volume $m$ for $m \leqslant m^{* *}$ (cf. [12, Theorem 1.3]). For the ball, the value $e_{0}(m)=$ $C_{1} m^{(d-1) / d}+C_{2} m^{(2 d-s) / d}$ is explicitly known (with universal constants $C_{1}, C_{2}$ ), and is strictly concave when $m<\widehat{m}:=\min \left\{m^{* *},\left(C_{1} / C_{2}\right)^{d /(1+d-s)}\right\}$. In particular, it follows that if the masses $\left\{m^{i}\right\}$ minimize the expression $e_{0}\left(M-m^{0}\right)=\sum_{i=1}^{\infty} e_{0}\left(m^{i}\right)$ then there can be at most one $m^{i} \in(0, \widehat{m})$. Hence, there can only be a finite number of components $E^{i}$.

This completes the proof of the concentration lemma.

The proof of Theorem 1 is essentially given in [3] for the Newtonian case $s=1$ and for more general confinement terms, but we include a short proof here for completeness.

Proof of Theorem 1. We apply Lemma 6 to any minimizing sequence $\left\{\Omega_{n}\right\}$ for $e_{Z}(M)$. If case (A) holds, the sequence converges to a minimizer and we are done. So assume there is splitting as in case (B), and so there exists $N \in \mathbb{N}$, sets $E^{i} \subset \mathbb{R}^{d}$ with 
$\left|E^{i}\right|=m^{i} \neq 0$ for each $i=0,1, \ldots, N, M=\sum_{i=0}^{N} m^{i}$, satisfying the lower bound,

$$
e_{Z}(M) \geqslant e_{Z}\left(m^{0}\right)+\sum_{i=1}^{N} e_{0}\left(m^{i}\right)
$$

We now construct a better upper bound, using the slow decay rate of the potential (recall that $0<p<s$ ). As each $E^{i}$ is a minimizer, it is essentially bounded (cf. [19, Lemma 4.1]). Hence, we may choose a representative for $E^{i}$ such that, for some $R>0$, we have $E^{i} \subset B_{R}(0)$ for all $i=0,1, \ldots, N$. For $i=1, \ldots, N$ let $b_{i} \in \mathbb{R}^{d}$ such that $\left|b_{i}\right|=1$, and let $b_{0}=0$. Define

$$
\Omega_{t}:=E^{0} \cup\left[\bigcup_{i=1}^{N}\left(E^{i}+t b_{i}\right)\right] .
$$

Note that for all sufficiently large $t$ the sets are disjoint, and so using the translation invariance of the perimeter and the nonlocal part $\mathcal{D}$, we have

$$
\begin{aligned}
e_{Z}(M) & \leqslant \mathbf{E}_{Z}\left(\Omega_{t}\right)=\mathbf{E}_{Z}\left(E^{0}\right)+\sum_{i=1}^{N} \mathbf{E}_{0}\left(E^{i}\right)+\mathbf{F}(t)-\mathbf{G}(t) \\
& =e_{Z}\left(m^{0}\right)+\sum_{i=1}^{N} e_{0}\left(m^{i}\right)+\mathbf{F}(t)-\mathbf{G}(t)
\end{aligned}
$$

where

$$
\mathbf{F}(t):=\sum_{\substack{i, j=0 \\ i \neq j}}^{N} \int_{E^{i}+t b_{i}} \int_{E^{j}+t b_{j}} \frac{d x d y}{|x-y|^{s}} \text { and } \mathbf{G}(t):=\sum_{i=1}^{N} \int_{E^{i}+t b_{i}} \frac{d x}{|x|^{p}}
$$

We now estimate each; first, we claim there is a $t_{0}>0$ for which $\mathbf{F}(t) \leqslant C t^{-s}$ for all $t>t_{0}$. Indeed, for any $i \neq j$, with the change of variables $t \xi=x, t \eta=y$, we have

$$
\begin{aligned}
t^{s} \int_{E^{i}+t b_{i}} \int_{E^{j}+t b_{j}} \frac{d x d y}{|x-y|^{s}} & \leqslant t^{s} \int_{B_{R}\left(t b_{i}\right)} \int_{B_{R}\left(t b_{j}\right)} \frac{d x d y}{|x-y|^{s}} \\
& =\frac{\left|B_{R}\right|^{2}}{\left|B_{R / t}\right|^{2}} \int_{B_{R / t}\left(b_{i}\right)} \int_{B_{R / t}\left(b_{j}\right)} \frac{d \xi d \eta}{|\xi-\eta|^{s}} \longrightarrow \frac{\left|B_{R}\right|^{2}}{\left|b_{i}-b_{j}\right|^{s}},
\end{aligned}
$$

as $t \rightarrow \infty$. There are only finitely many terms in $\mathbf{F}(t)$, and so the claim holds.

To estimate $\mathbf{G}(t)$ from below, we note that as $t \rightarrow \infty$,

$$
t^{-p} \int_{E^{i}+t b_{i}}|x|^{-p} d x=\int_{E^{i}}\left|b_{i}+\frac{x}{t}\right|^{-p} d x \longrightarrow\left|E^{i}\right|=m^{i},
$$

by dominated convergence. Thus, $\mathbf{F}(t)-\mathbf{G}(t) \leqslant c_{1} t^{-s}-M Z t^{-p}<0$ for sufficiently large $t$, and thus (27) is in contradiction with (26). Thus we must have $\left|\Omega^{0}\right|=M$ and $e_{Z}(M)=\mathbf{E}_{Z}\left(E^{0}\right)$, for any $M>0$ and for any $Z>0$. 
DROPLET BREAKUP IN THE LIQUID DROP MODEL WITH BACKGROUND POTENTIAL13

\section{The Limit $Z \rightarrow 0$}

We start this section by proving that the finite dimensional energy functional $\mathbf{F}_{N, \underline{m}}$ given by (6) has a minimizer. We define

$$
\mu_{N, \underline{m}}:=\inf _{\Sigma_{N}} \mathbf{F}_{N, \underline{m}},
$$

where the admissible set $\Sigma_{N}$ is defined in (7).

Proposition 8. For any $N \in \mathbb{N}$ and $\underline{m}$, the functional $\mathbf{F}_{N, \underline{m}}$ attains its minimum $\mu_{N, \underline{m}}<0$ on the admissible class $\Sigma_{N}$.

Proof. Consider any minimizing sequence $\left\{x_{n}^{i}\right\}_{n \in \mathbb{N}}, i=1, \ldots, N$, in $\Sigma_{N}$, that is, $\mu_{N, \underline{m}}=\lim _{n \rightarrow \infty} \mathbf{F}_{N, \underline{m}}\left(0, x_{1}^{n}, \ldots, x_{N}^{n}\right)$. If all the sequences $\left\{x_{n}^{i}\right\}_{n \in \mathbb{N}}, i=1, \ldots, N$, remain bounded, then we obtain convergence to a minimizer along some subsequence. So instead, assume that there is an integer $k \in\{0,1, \ldots, N-1\}$ and a subsequence (not relabelled) so that

$$
\left\{\begin{array}{r}
x_{n}^{i} \underset{n \rightarrow \infty}{\longrightarrow} a_{i}, \quad \forall i=0, \ldots, k, \text { but } \\
\left|x_{n}^{i}\right| \underset{n \rightarrow \infty}{\longrightarrow} \infty, \quad \forall i=(k+1), \ldots, N .
\end{array}\right.
$$

We first treat the case where $k \geqslant 1$. Decompose $\mathbf{F}_{N, \underline{m}}$ into pieces,

$$
\begin{aligned}
\mathbf{F}_{N, \underline{m}}\left(0, x_{n}^{1}, \ldots, x_{n}^{N}\right)=\mathbf{F}_{k,\left(m^{0}, \ldots, m^{k}\right)} & \left(0, x_{n}^{1}, \ldots, x_{n}^{k}\right) \\
& +\mathbf{F}_{N-k,\left(m^{k+1}, \ldots, m^{N}\right)}\left(x_{n}^{k+1}, \ldots, x_{n}^{N}\right)+I_{k, N},
\end{aligned}
$$

with interaction term between the two families,

$$
I_{k, N}=\sum_{i=0}^{k} \sum_{j=k+1}^{N} \frac{m^{i} m^{j}}{\left|x_{n}^{i}-x_{n}^{j}\right|^{s}} .
$$

Using the splitting (28), we have

$$
\begin{aligned}
\mu_{N, \underline{m}} & \geqslant \liminf _{n \rightarrow \infty}\left[\mathbf{F}_{k,\left(m^{0}, \ldots, m^{k}\right)}\left(0, x_{n}^{1}, \ldots, x_{n}^{k}\right)+\sum_{\substack{i, j=k+1 \\
j \neq i}}^{N} \frac{m^{i} m^{j}}{\left|x_{n}^{i}-x_{n}^{j}\right|^{s}}\right] \\
& \geqslant \liminf _{n \rightarrow \infty} \mathbf{F}_{k,\left(m^{0}, \ldots, m^{k}\right)}\left(0, x_{n}^{1}, \ldots, x_{n}^{k}\right) \\
& =\mathbf{F}_{k,\left(m^{0}, \ldots, m^{k}\right)}\left(0, a_{1}, \ldots, a_{k}\right) .
\end{aligned}
$$

To obtain a contradiction to (30), we define a new configuration given by the points $\left\{a_{1}, \ldots, a_{k}, R y_{1}, \ldots, R y_{N-k}\right\}$ with $\left\{y_{1}, \ldots, y_{N-k}\right\}$ distinct points on the unit sphere $\left|y_{j}\right|=1$, and $R>0$ to be determined. By the same decomposition as in (29),

$$
\begin{array}{r}
\mathbf{F}_{N, \underline{m}}\left(0, a_{1}, \ldots, a_{k}, R y_{1}, \ldots, R y_{N-k}\right)=\mathbf{F}_{k,\left(m^{0}, \ldots, m^{k}\right)}\left(0, a_{1}, \ldots, a_{k}\right) \\
+\mathbf{F}_{N-k,\left(m^{k+1}, \ldots, m^{N}\right)}\left(R y_{1}, \ldots, R y_{N-k}\right)+\tilde{I}_{k, N}
\end{array}
$$


with $\tilde{I}_{k, N}$ representing the interaction terms. If $\left|a_{i}\right|<R_{0}$ for some $R_{0}>0$ and for each $i=1, \ldots, k$, and if $R>2 R_{0}$, the interaction terms may be estimated by

$$
\tilde{I}_{k, N} \leqslant C_{1}(k, N, \underline{m}) R^{-s} .
$$

Similarly, since $\left|R y_{i}-R y_{j}\right| \geqslant C_{2} R, i \neq j$, for some constant $C_{2}>0$, we also have

$$
\mathbf{F}_{N-k,\left(m^{k+1}, \ldots, m^{N}\right)}\left(R y_{1}, \ldots, R y_{N-k}\right) \leqslant \sum_{\substack{i, j=1 \\ i \neq j}}^{N-k} \frac{m^{k+i} m^{k+j}}{\left|R y_{i}-R y_{j}\right|^{s}} \leqslant C_{3}(k, N, \underline{m}) R^{-s} .
$$

On the other hand,

$$
\sum_{i=1}^{N-k} m^{k+i}\left|R y_{i}\right|^{-p}=R^{-p} \sum_{i=1}^{N-k} m^{k+i} \geqslant C_{4}(k, N, \underline{m}) R^{-p} .
$$

and thus (31) yields,

$$
\begin{aligned}
\mu_{N, \underline{m}} & \leqslant \mathbf{F}_{N, \underline{m}}\left(0, a_{1}, \ldots, a_{k}, R y_{1}, \ldots, R y_{N-k}\right) \\
& \leqslant \mathbf{F}_{k,\left(m^{0}, \ldots, m^{k}\right)}\left(0, a_{1}, \ldots, a_{k}\right)-C_{4}(k, N, \underline{m}) R^{-p}+O\left(R^{-s}\right) \\
& <\mathbf{F}_{k,\left(m^{0}, \ldots, m^{k}\right)}\left(0, a_{1}, \ldots, a_{k}\right),
\end{aligned}
$$

for $R>R_{0}>0$ chosen large enough, contradicting (30) in case $k \geqslant 1$. For $k=0$, that is, if $\left|x_{n}^{i}\right| \rightarrow \infty$ for each $i=1, \ldots, N$, we note that

$$
\mu_{N, \underline{m}} \geqslant \liminf _{n \rightarrow \infty} \sum_{\substack{i, j=0 \\ j \neq i}}^{N} \frac{m^{i} m^{j}}{\left|x_{n}^{i}-x_{n}^{j}\right|^{s}} \geqslant 0
$$

while the same construction which produced (32) yields the contradictory estimate $\mu_{N, \underline{m}}<0$. In conclusion, the entire minimizing sequence must remain bounded, and so the minimum is attained.

Next we show that the infimum of the regularized energies $\mathbf{E}_{Z}$ converge to the infimum of $\mathbf{E}_{0}$.

Lemma 9. $\lim _{Z \rightarrow 0} e_{Z}(M)=e_{0}(M)$.

Proof. Let $\Omega_{Z}$ be a minimizer of $\mathbf{E}_{Z}$ which exists for any $Z>0$ and $M>0$ by Theorem 1. Then, clearly $e_{Z}(M) \leqslant e_{0}(M)$ for all $Z>0$, and

$$
\begin{aligned}
\mathbf{E}_{0}\left(\Omega_{Z}\right)= & \mathbf{E}_{Z}\left(\Omega_{Z}\right)+Z \int_{\Omega_{Z}} \frac{d x}{|x|^{p}} \leqslant \mathbf{E}_{Z}\left(\Omega_{Z}\right)+Z \int_{B_{1}(0)} \frac{d x}{|x|^{p}} \\
& \quad+Z\left|\Omega_{Z} \cap\left(\mathbb{R}^{d} \backslash B_{1}(0)\right)\right| \\
\leqslant & \mathbf{E}_{Z}\left(\Omega_{Z}\right)+\left(\frac{\omega_{d}}{(d-p)}+M\right) Z,
\end{aligned}
$$


DROPLET BREAKUP IN THE LIQUID DROP MODEL WITH BACKGROUND POTENTIAL15

where $\omega_{d}=\left|B_{1}(0)\right|$ denotes the volume of the unit ball in $\mathbb{R}^{d}$. Therefore we also have $e_{0}(M) \leqslant \liminf _{Z \rightarrow 0} e_{Z}(M)$, which proves the claim.

The following lemma is key in obtaining regularity properties for a family of minimizers of the functionals $\mathbf{E}_{Z}$.

Lemma 10. The family of minimizers $\left\{\Omega_{Z}\right\}_{Z \in(0,1]}$ of $\mathbf{E}_{Z}$ are $(\omega, r)$-minimizers of the perimeter functional in $\mathcal{O}:=\mathbb{R}^{d} \backslash \overline{B_{\delta}(0)}$ for any $\delta>0$, with $\omega, r>0$ uniformly chosen for $Z \in(0,1]$; that is,

$$
\operatorname{Per}\left(\Omega_{Z}\right) \leqslant \operatorname{Per}(F)+\omega\left|\Omega_{Z} \triangle F\right|,
$$

for all $F \subset \mathbb{R}^{d}$ with $\Omega_{Z} \triangle F \subset B_{r}\left(x_{0}\right) \subset \mathbb{R}^{d} \backslash B_{\delta}(0)$.

Proof. First we show that the constraint $\left|\Omega_{Z}\right|=M$ may be replaced by a penalization, following [6, Theorem 2.7] (see also [11, Section 2].) For $\lambda>0$ (to be determined), define the penalized functionals

$$
\mathcal{F}_{Z}^{\lambda}(F):=\mathbf{E}_{Z}(F)+\lambda|| F|-| \Omega_{Z}||=\mathbf{E}_{Z}(F)+\lambda|| F|-M| .
$$

We claim that there exists $\lambda>0$ so that for all $0<Z \leqslant 1$,

$$
\min \mathcal{F}_{Z}^{\lambda}=\mathcal{F}_{Z}^{\lambda}\left(\Omega_{Z}\right)=\mathbf{E}_{Z}\left(\Omega_{Z}\right),
$$

i.e., the unconstrained minimizer of $\mathcal{F}_{Z}^{\lambda}$ coincides with the mass-constrained minimizer of $\mathbf{E}_{Z}$. Indeed, the existence of a constant $\lambda=\lambda_{Z}>0$ for each fixed $Z>0$ satisfying the claim follows by a minor modification of [․․, Theorem 2.7], so it suffices to show that $\lambda$ may be chosen independently of $Z$. Suppose no such $\lambda$ exists, so there are sequences $Z_{n} \rightarrow 0, \lambda_{n} \rightarrow \infty$, and sets $E_{n} \subset \mathbb{R}^{d},\left|E_{n}\right| \neq M$, with $\mathcal{F}_{Z_{n}}^{\lambda_{n}}\left(E_{n}\right)<\mathcal{F}_{Z_{n}}^{\lambda_{n}}\left(\Omega_{Z_{n}}\right)$. Note that $\lambda_{n} \rightarrow \infty$ implies that $\left|E_{n}\right| \rightarrow M$.

Define sets $\tilde{E}_{n}=t_{n} E_{n}$ where $t_{n}=\left[M /\left|E_{n}\right|\right]^{1 / d}$, so $\left|\tilde{E}_{n}\right|=M$. Each term in $\mathcal{F}_{Z_{n}}\left(\tilde{E}_{n}\right)$ may then be calculated via scaling,

$$
\begin{aligned}
\mathcal{F}_{Z_{n}}^{\lambda_{n}}\left(\tilde{E}_{n}\right)= & \mathbf{E}_{Z_{n}}\left(\tilde{E}_{n}\right)=t_{n}^{d-1} \operatorname{Per}\left(E_{n}\right)+t_{n}^{2 d-s} \mathcal{D}\left(E_{n}, E_{n}\right)-t_{n}^{d-p} Z_{n} \int_{E_{n}}|x|^{-p} d x \\
= & \mathcal{F}_{Z_{n}}^{\lambda_{n}}\left(E_{n}\right)+\left(t_{n}^{d-1}-1\right) \operatorname{Per}\left(E_{n}\right)+\left(t_{n}^{2 d-s}-1\right) \mathcal{D}\left(E_{n}, E_{n}\right) \\
& \quad-\left(t_{n}^{d-p}-1\right) Z_{n} \int_{E_{n}}|x|^{-p} d x-\lambda_{n}\left|t_{n}^{d-1}-1\right|\left|E_{n}\right| \\
\leqslant & \mathcal{F}_{Z_{n}}^{\lambda_{n}}\left(E_{n}\right)+\left|t_{n}^{d-1}-1\right|\left|E_{n}\right|\left[\mathbf{E}_{0}\left(E_{n}\right) \frac{\left(t_{n}^{d-1}+t_{n}^{2 d-s}-2\right)}{\left|t_{n}^{d-1}-1\right|\left|E_{n}\right|}-\lambda_{n}\right] \\
< & \mathcal{F}_{Z_{n}}^{\lambda_{n}}\left(E_{n}\right),
\end{aligned}
$$

as $\lambda_{n} \rightarrow \infty$ since the term in brackets is eventually negative. This contradicts the definition of $E_{n}$ as minimizers of $\mathcal{F}_{Z_{n}}^{\lambda_{n}}$, and so we conclude that (33) must hold. 
Now fix any $r>0$ and assume $B_{r}\left(x_{0}\right) \cap B_{\delta}(0)=\emptyset$, and $F \subset \mathbb{R}^{d}$ with $\Omega_{Z} \triangle F \subset$ $B_{r}\left(x_{0}\right)$. Denote

$$
\mathcal{V}(F):=\int_{F} \frac{d x}{|x|^{p}} .
$$

Then, $\mathbf{E}_{Z}\left(\Omega_{Z}\right)=\mathcal{F}_{Z}^{\lambda}\left(\Omega_{Z}\right) \leqslant \mathcal{F}_{Z}^{\lambda}(F)$ implies that

$$
\begin{aligned}
\operatorname{Per}\left(\Omega_{Z}\right) & \leqslant \operatorname{Per}(F)+\left(\mathcal{D}(F, F)-\mathcal{D}\left(\Omega_{Z}, \Omega_{Z}\right)\right)+\left(\mathcal{V}\left(\Omega_{Z}\right)-\mathcal{V}(F)\right)+\lambda|| F|-M| \\
& \leqslant \operatorname{Per}(F)+\left(C_{0}+\delta^{-p}+\lambda\right)\left|\Omega_{Z} \triangle F\right|,
\end{aligned}
$$

where the difference of the nonlocal terms is estimated in [6, Proposition 2.3], and to estimate the confinement term we use the fact that $|x|^{-p} \in L^{\infty}\left(\mathbb{R}^{d} \backslash B_{\delta}(0)\right)$. Thus, $\Omega_{Z}$ are $(\omega, r)$-minimizers of the perimeter functional in $\mathbb{R}^{d} \backslash B_{\delta}(0)$ with $\omega=C_{0}+\delta^{-p}+\lambda$ and any $r>0$.

Finally, we state the following regularity results for $(\omega, r)$-minimizers that we will require in the proof of Theorems 2 and 5 ,

Lemma 11 (see Theorems 21.8, 21.14 and 26.6 of [26]). Let $\mathcal{O} \subset \mathbb{R}^{d}$ be an open set.

(i) If $E \subset \mathbb{R}^{d}$ is an $(\omega, r)$-minimizer of perimeter in $\mathcal{O}$ then $\partial^{*} E \cap \mathcal{O}$ is a $C^{1, \alpha}$ hypersurface for any $\alpha \in(0,1 / 2)$.

(ii) If $E_{n} \subset \mathbb{R}^{d}$ is a sequence of uniformly $(\omega, r)$-minimizers of perimeter in $\mathcal{O}$ with $E_{n} \rightarrow E_{\infty}$ locally in $\mathcal{O}$, then for any sequence $x_{n} \in \partial E_{n}$ with $x_{n} \rightarrow x_{\infty}$ we have $x_{\infty} \in \partial E_{\infty}$. Moreover, if $x_{n} \in \partial^{*} E_{n}$, then $x_{\infty} \in \partial^{*} E_{\infty}$ and the normal vectors satisfy $\nu\left(x_{n}\right) \rightarrow \nu\left(x_{\infty}\right)$.

Thus, a sequence of uniformly $(\omega, r)$-minimizers of perimeter which converges locally has its reduced boundary convergent in the Hausdorff metric. We remark that a stronger form of this $C^{1, \alpha}$ convergence of $\partial^{*} E_{n} \rightarrow \partial^{*} E$ is stated in [1, Theorem 4.2]: under the hypothesis that $E_{n} \rightarrow E$ globally in $\mathcal{O}$, in fact the convergence of the boundaries is in $C^{1, \alpha}$ for $\alpha \in(0,1 / 2)$, and $\partial E_{n}$ may be realized as a $C^{1, \alpha}$ graph over $\partial E$.

We remark that we only need the full force of the regularity theory for Theorem 5 , For the proof of Theorem 2 we only require that minimizers for $\mathbf{E}_{Z}$ are supported in compact sets and converge pointwise to the disjoint components $E^{i}$.

Now we are ready to prove our main results.

Proof of Theorem 圆. Let $\left\{\Omega_{n}\right\}_{n \in \mathbb{N}}$ with $\Omega_{n}:=\Omega_{Z_{n}}$ be a sequence of minimizers for $e_{Z_{n}}$ with $Z_{n} \rightarrow 0$. By Lemma 9, $\left\{\Omega_{n}\right\}$ form in fact a minimizing sequence for $e_{0}$. Therefore by Lemma 6 we obtain either (A) or assertions (i), (ii), and (9), (10) in (iii) of part (B) of Theorem 2, The statement (8), on the other hand, follows directly 
from Lemmas 10 and 11 (or [1, Theorem 4.2].) In order to prove (11) we adopt the notations from Lemma 6. Our goal here is to use the regularity of minimizing sets to improve the precision of the lower bound defined in the concentration lemma. We prove this in several steps.

Step 1: A more refined decomposition. We return to Step 1 in the proof of Lemma 6, and use the uniform $(\omega, r)$-minimality to show that

$$
\Omega_{n}=F_{n}^{0} \cup\left[\bigcup_{i=1}^{k}\left(F_{n}^{i}+x_{n}^{i}\right)\right],
$$

splits cleanly, with no $o(1)$ error in the perimeter, and with remainder set $\Omega_{n}^{N}=\emptyset$. In particular, we claim that

$$
\operatorname{Per}\left(\Omega_{n}\right)=\sum_{i=0}^{N} \operatorname{Per}\left(F_{n}^{i}\right)
$$

holds for each sufficiently large $n$. For convenience, we define

$$
\tilde{F}_{n}^{i}=F_{n}^{i}+x_{n}^{i} \quad \text { and } \quad \hat{\Omega}_{n}^{i}=\Omega_{n}^{i-1}-x_{n}^{i}, \quad i=0,1, \ldots, N .
$$

To verify (34), we first note that $E^{i}$ being minimizers of $e_{0}\left(m^{i}\right)$, they are essentially bounded domains with smooth $\partial^{*} E^{i}$ (cf. [19, Proposition 2.1 and Lemma 4.1]). Therefore, we may fix $R>0$ so that a representative of each $E^{i} \subset B_{R / 2}(0)$ for each $i=0,1, \ldots, N$. We observe that, since each $E^{i}$ is bounded, when defining $F_{n}^{i}=\hat{\Omega}_{n}^{i-1} \cap B_{r_{n}}(0)$ we may choose the radii $r_{n}$ found in [15, Lemma 2.2] so that $r_{n} \in(R, 2 R)$. As $\hat{\Omega}_{n}^{i} \rightarrow E^{i}$ locally, it converges globally in $\mathcal{O}:=B_{2 R}(0)$. For $i=1, \ldots, N$, we invoke Lemma 10 which ensures that $\hat{\Omega}_{n}^{i}$ is a family of uniformly $(\omega, r)$-minimizers in $\mathcal{O}$. By part (ii) the regularity result Lemma 11, $\hat{\Omega}_{n}^{i} \cap \mathcal{O} \rightarrow$ $E^{i} \subset B_{R / 2}(0)$ in Hausdorff norm, so in particular $\hat{\Omega}_{n}^{i} \cap B_{2 R}(0) \subset B_{R}(0)$ for all sufficiently large $n$. When $i=0$ there is the slightly delicate issue that $\Omega_{n}$ are not necessarily $(\omega, r)$-minimizers in a neighborhood of 0 . For $i=0$, define the open set $\tilde{\mathcal{O}}:=B_{2 R}(0) \backslash \overline{B_{\delta}(0)}$, with any $\delta \in(0, R / 2)$, so $\Omega_{n}$ are uniformly $(\omega, r)$-minimizers in $\tilde{\mathcal{O}}$. Again, by part (ii) of Lemma 11 we conclude that $\Omega_{n} \cap\left[B_{2 R}(0) \backslash B_{R}(0)\right]=\emptyset$ for all sufficiently large $n$.

Finally, suppose $\Omega_{n}^{N} \neq \emptyset$ for all $n \in \mathbb{N}$. Recall that by Lemma 6 , $\left|\Omega_{n}^{N}\right| \rightarrow 0$, so $\Omega_{n}^{N} \rightarrow \emptyset$ globally. As $\Omega_{n}$ is an $(\omega, r)$-minimizing sequence each $\partial^{*} \Omega_{n}^{N}$ is a smooth hypersurface, and there would then exist $y_{n} \in \partial \Omega_{n}^{N}$ for each $n$. The translates $\hat{\Omega}_{n}^{N}:=\Omega_{n}^{N}-y_{n}$ are again smooth, with $0 \in \partial \hat{\Omega}_{n}^{N}$ for each $n$. Invoking (ii) of Lemma 11 we arrive at a contradiction, because then 0 lies on the boundary of the limit set of the $\hat{\Omega}_{n}^{N}$, which is empty. Therefore we must have $\Omega_{n}^{N}=\emptyset$ for large $n$.

As $\left|x_{n}^{i}-x_{n}^{j}\right| \rightarrow \infty$ for $i \neq j$, and each $G_{n}^{i} \cap B_{R}(0)=\emptyset$, the components are well separated, and we obtain (34). 
We remark that (34) also implies the equality of masses before and after passing to the limit, that is:

$$
M=\sum_{i=1}^{N} m^{i}=\sum_{i=1}^{N} m_{n}^{i}
$$

holds (with no error) for all $n$ sufficiently large.

Step 2: $\quad E^{0} \neq \emptyset$. Suppose the contrary. Since there are only finitely many components, we may choose $k \in\{1,2, \ldots, N\}$ and a subsequence (not relabelled) along which we have $\left|x_{n}^{k}\right|=\min \left\{\left|x_{n}^{j}\right|: j=1, \ldots, N\right\}$. Consider the sets $\breve{\Omega}_{n}:=$ $\Omega_{n}-x_{n}^{k}$. The perimeter and nonlocal terms in $\mathbf{E}_{Z}$ are translation invariant, hence, this modification only affects the confinement term $\mathcal{V}$. By Step 3, we have a disjoint decomposition,

$$
\breve{\Omega}_{n}=F_{n}^{0} \cup F_{n}^{k} \cup\left[\bigcup_{\substack{i=1 \\ i \neq k}}^{N}\left(F_{n}^{i}+y_{n}^{i}\right)\right] \cup \Omega_{n}^{N},
$$

where $y_{n}^{i}=x_{n}^{i}-x_{n}^{k}$, with $\left|y_{n}^{i}\right| \rightarrow \infty, i \neq k$. Therefore, $\mathcal{V}\left(F_{n}^{j}+x_{n}^{j}\right) \rightarrow 0$ and $\mathcal{V}\left(F_{n}^{i}+y_{n}^{i}\right) \rightarrow 0$, for all $j=1, \ldots, N$ and for all $i \neq k$, while $\mathcal{V}\left(F_{n}^{k}\right) \rightarrow \mathcal{V}\left(E^{k}\right)>0$. Hence,

$$
\begin{aligned}
\mathbf{E}_{Z_{n}}\left(\breve{\Omega}_{n}\right)-\mathbf{E}_{Z_{n}}\left(\Omega_{n}\right) & =-Z_{n} \mathcal{V}\left(F_{n}^{k}\right)-Z_{n} \sum_{\substack{i=1 \\
i \neq k}}^{N} \mathcal{V}\left(F_{n}^{i}+y_{n}^{i}\right)+Z_{n} \sum_{i=1}^{N} \mathcal{V}\left(F_{n}^{i}+x_{n}^{i}\right) \\
& =-Z_{n} \mathcal{V}\left(E^{k}\right)+o\left(Z_{n}\right)<0,
\end{aligned}
$$

which contradicts the minimality of $\Omega_{n}$. Hence we must have $\left|E^{0}\right| \neq 0$.

Step 3: A more refined lower bound. As in Step 1, there exists $R>0$ for which $F_{n}^{i} \subset B_{R}(0)$ for each $n \in \mathbb{N}$ and $i=0,1, \ldots, N$. Since $\bigcup_{i=0}^{N}\left(F_{n}^{i}+x_{n}^{i}\right) \subset \Omega_{n}$, we may decompose the nonlocal term and obtain

$$
\mathcal{D}\left(\Omega_{n}, \Omega_{n}\right) \geqslant \sum_{i, j=0}^{N} \mathcal{D}\left(\tilde{F}_{n}^{i}, \tilde{F}_{n}^{j}\right)
$$

Let

$$
R_{n, i j}:=\left|x_{n}^{i}-x_{n}^{j}\right| \quad \text { and } \quad R_{n, i 0}:=\left|x_{n}^{i}\right| .
$$

Then, for all $x \in \tilde{F}_{n}^{i}, y \in \tilde{F}_{n}^{j}$ and sufficiently large $n$, we have

$$
|x-y| \geqslant R_{n, i j}-2 R \geqslant \frac{1}{2} R_{n, i j} .
$$

By the mean value theorem for $f(t)=t^{s}$ we then calculate,

$$
|| x_{n}^{i}-\left.x_{n}^{j}\right|^{s}-|x-y|^{s}\left|\leqslant s\left(\frac{1}{2} R_{n, i j}\right)^{s-1}\right| x_{n}^{i}-x_{n}^{j}-x+y \mid
$$


DROPLET BREAKUP IN THE LIQUID DROP MODEL WITH BACKGROUND POTENTIAL 19

$$
\leqslant C R_{n, i j}^{s-1}\left(\left|x_{n}^{i}-x\right|+\left|x_{n}^{j}-y\right|\right) \leqslant 2 C R R_{n, i j}^{s-1}
$$

Hence, for all sufficiently large $n$,

$$
\left|\frac{1}{|x-y|^{s}}-\frac{1}{\left|x_{n}^{i}-x_{n}^{j}\right|^{s}}\right|=\frac{|| x_{n}^{i}-\left.x_{n}^{j}\right|^{s}-|x-y|^{s} \mid}{|x-y|^{s}\left|x_{n}^{i}-x_{n}^{j}\right|^{s}} \leqslant \frac{C}{R_{n, i j}^{s+1}}
$$

for all $0<s<d$, and we may estimate the off-diagonal terms in the nonlocal energy via

$$
\begin{aligned}
\left|\mathcal{D}\left(\tilde{F}_{n}^{i}, \tilde{F}_{n}^{j}\right)-\frac{m_{n}^{i} m_{n}^{j}}{\left|x_{n}^{i}-x_{n}^{j}\right|^{s}}\right| & \\
& \leqslant \int_{\tilde{F}_{n}^{i}} \int_{\tilde{F}_{n}^{j}}\left|\frac{1}{|x-y|^{s}}-\frac{1}{\left|x_{n}^{i}-x_{n}^{j}\right|^{s}}\right| d x d y \leqslant C R_{n, i j}^{-s-1},
\end{aligned}
$$

with a constant $C$ independent of $n$.

The confinement term may be evaluated in a similar way: we have

$$
\left.|| x_{n}^{i}\right|^{-p}-\left.|x|^{-p}\left|\leqslant \sup _{\xi \in \tilde{F}_{n}^{i}} p\right| \xi\right|^{-p-1}\left|x-x_{n}^{i}\right| \leqslant C\left|x_{n}^{i}\right|^{-p-1} \leqslant C R_{n, i 0}^{-p-1}
$$

and thus

$$
\left|\int_{\tilde{F}_{n}^{i}} \frac{d x}{|x|^{p}}-\frac{m_{n}^{i}}{\left|x_{n}^{i}\right|^{p}}\right| \leqslant C R_{n, i 0}^{-p-1} .
$$

Putting the above estimates together with the perimeter splitting (34), we obtain a lower bound,

$$
\begin{aligned}
\mathbf{E}_{Z_{n}}\left(\Omega_{n}\right) \geqslant & \sum_{i=0}^{N} \mathbf{E}_{0}\left(F_{n}^{i}\right)-Z_{n} \mathcal{V}\left(F_{n}^{0}\right)+\sum_{\substack{i, j=0 \\
i \neq j}}^{N} \frac{m_{n}^{i} m_{n}^{j}}{\left|x_{n}^{i}-x_{n}^{j}\right|^{s}}\left(1-O\left(R_{n, i j}^{-1}\right)\right) \\
& -Z_{n} \sum_{i=1}^{N} \frac{m_{n}^{i}}{\left|x_{n}^{i}\right|^{p}}\left(1+O\left(R_{n, i 0}^{-1}\right)\right) \\
\geqslant & \sum_{i=0}^{N} e_{0}\left(m_{n}^{i}\right)-Z_{n} \mathcal{V}\left(F_{n}^{0}\right)+\sum_{\substack{i, j=0 \\
i \neq j}}^{N} \frac{m_{n}^{i} m_{n}^{j}}{\left|x_{n}^{i}-x_{n}^{j}\right|^{s}}\left(1-O\left(R_{n, i j}^{-1}\right)\right) \\
\geqslant & \sum_{i=0}^{N} e_{0}\left(m_{n}^{i}\right)-Z_{n} \mathcal{V}\left(F_{n}^{0}\right)+\sum_{\substack{i, j=0 \\
i \neq j}}^{N} \frac{m^{i} m^{j}}{\left|x_{n}^{i}-x_{n}^{j}\right|^{s}}(1-o(1)) \\
(38) & -\sum_{i=1}^{N} \frac{m_{n}^{i}}{\left|x_{n}^{i}\right|^{p}}\left(1+O\left(R_{n, i 0}^{-1}\right)\right) \\
& -Z_{n} \sum_{i=1}^{N} \frac{m^{i}}{\left|x_{n}^{i}\right|^{p}}(1+o(1)),
\end{aligned}
$$


where in the last line we have used the convergence $m_{n}^{i} \rightarrow m^{i}$.

Step 4: A more refined upper bound. In order to obtain a more refined upper bound, let $\Omega_{t}=F_{n}^{0} \cup\left[\bigcup_{i=1}^{N}\left(F_{n}^{i}+t a^{i}\right)\right]$, with sets $F_{n}^{i}$ as in Lemma 6, with points $\left\{a^{i}\right\}_{i=1, \ldots, N} \subset \mathbb{R}^{d}$ with $0<\left|a^{i}\right| \leqslant 1$, and $t>0$ is to be determined optimally. Substituting $\Omega_{t}$ into $\mathbf{E}_{Z}$ we recover an upper bound of the same general form as (27) as before,

$$
\begin{aligned}
e_{Z_{n}}(M) \leqslant & \mathbf{E}_{Z_{n}}\left(\Omega_{t}\right) \\
\leqslant & \sum_{i=0}^{N} e_{0}\left(m_{n}^{i}\right)-Z_{n} \mathcal{V}\left(F_{n}^{0}\right)+\sum_{\substack{i, j=0 \\
i \neq j}}^{N} \int_{F_{n}^{i}+t a^{i}} \int_{F_{n}^{j}+t a^{j}} \frac{d x d y}{|x-y|^{s}} \\
& \quad-Z_{n} \sum_{i=1}^{N} \int_{F_{n}^{i}+t a^{i}}|x|^{-p} d x
\end{aligned}
$$

By the same estimates (36) and (37) as in Step 3 above, we thus have

$$
\left|\mathcal{D}\left(F_{n}^{i}, F_{n}^{j}\right)-\frac{m^{i} m^{j}}{t^{s}\left|a^{i}-a^{j}\right|^{s}}\right| \leqslant C t^{-s-1}, \quad\left|\int_{\tilde{F}_{n}^{i}} \frac{d x}{|x|^{p}}-\frac{m^{i}}{t^{p}\left|a^{i}\right|^{p}}\right| \leqslant C t^{-p-1},
$$

for constant $C$ independent of $t$. Choosing $t=t_{n}:=Z_{n}^{-1 /(s-p)}$, we then obtain the upper bound of the form:

$$
\begin{aligned}
e_{Z_{n}}(M) \leqslant \mathbf{E}_{Z_{n}}\left(\Omega_{t_{n}}\right) \leqslant & \sum_{i=0}^{N} e_{0}\left(m_{n}^{i}\right)-Z_{n} \mathcal{V}\left(F_{n}^{0}\right) \\
& \quad+Z_{n}^{s /(s-p)} \mathbf{F}_{N, \underline{m}}\left(0, a^{1}, \ldots, a^{N}\right)+O\left(Z_{n}^{\frac{s+1}{s-p}}\right) .
\end{aligned}
$$

By Proposition 8 , we may choose $\left(a^{1}, \ldots, a^{N}\right)$ to minimize $\mathbf{F}_{N, \underline{m}}$, and thus obtain the best upper bound,

$$
\mathbf{E}_{Z_{n}}\left(\Omega_{t_{n}}\right) \leqslant \sum_{i=0}^{N} e_{0}\left(m_{n}^{i}\right)-Z_{n} \mathcal{V}\left(F_{n}^{0}\right)+Z_{n}^{s /(s-p)} \mu_{N, \underline{m}}+o\left(Z_{n}^{s /(s-p)}\right) .
$$

Step 5: The scale of $x_{n}^{i}=O\left(Z^{-1 /(s-p)}\right)$. Lastly, we prove (11). To this end, let $\xi_{n}^{i}=x_{n}^{i} Z_{n}^{1 /(s-p)}$ for $i=1, \ldots, N$. Using the upper bound (39) followed by the lower bound (38) we find

$$
\begin{aligned}
Z_{n}^{s /(s-p)} \mu_{N, \underline{m}}+o\left(Z_{n}^{s /(s-p)}\right) & \geqslant \mathbf{E}_{Z_{n}}\left(\Omega_{n}\right)-\sum_{i=0}^{N} e_{0}\left(m_{n}^{i}\right)+Z_{n} \mathcal{V}\left(F_{n}^{0}\right) \\
& \geqslant Z_{n}^{s /(s-p)} \mathbf{F}_{N, \underline{m}}\left(0, \xi_{n}^{1}, \ldots, \xi_{n}^{N}\right)(1+o(1)) .
\end{aligned}
$$

After dividing by $Z_{n}^{s /(s-p)}$, we conclude that $\left\{\xi_{n}^{i}\right\}_{i=0, \ldots, N}$ is a minimizing sequence for $\mathbf{F}_{N, \underline{m}}$; by Proposition 8 , the $\xi_{n}^{i}$ are in fact bounded, and up to the extraction of 
DROPLET BREAKUP IN THE LIQUID DROP MODEL WITH BACKGROUND POTENTIAL21

a subsequence for each $i=1, \ldots, N, \xi_{n}^{i} \rightarrow y^{i}$, minimizers of $\mathbf{F}_{N, \underline{m}}$, as $n \rightarrow \infty$. We thus obtain (11), and the proof of Theorem 2 is complete.

Remark 12. We note that the proof of (11) in Step 5 above also shows that we have an expansion of the minimizing energy accurate up to the third-order term, namely,

$$
\mathbf{E}_{Z_{n}}\left(\Omega_{n}\right)=\sum_{i=0}^{N} e_{0}\left(m^{i}\right)-Z_{n} \mathcal{V}\left(F_{n}^{0}\right)+Z_{n}^{\frac{s}{s-p}} \mathbf{F}_{N, \underline{m}}\left(0, y_{1}, \ldots, y_{N}\right)+o\left(Z_{n}^{\frac{s}{s-p}}\right),
$$

where $F_{n}^{0}$ are the sets constructed in Lemma 6. One might be tempted to pass to the limit $F_{n}^{i} \rightarrow E^{i}$ and express the expansion in terms of the components of the generalized minimizer, but it is not at all clear what the error term in such an expansion would be.

Finally, we prove Theorem 5 .

Proof of Theorem 5. By Step 1 in the proof of Theorem 2 and 11 (see also Theorem 27.5 of [26]) the reduced boundary $\partial^{*} \Omega_{n}$ is a disjoint union of smooth hypersurfaces. In fact, by [6, Theorem 2.7], $\partial^{*} \Omega_{n}$ is of class $C^{3, \beta}$ for $\beta<d-1-s$. In particular, the Euler-Lagrange equation,

$$
(d-1) \kappa(x)+2 v_{\Omega_{n}}(x)-Z_{n}|x|^{-p}=\lambda_{n},
$$

is satisfied pointwise on $\partial^{*} \Omega_{n}$, where $\kappa$ is the mean curvature in $\mathbb{R}^{d}, \lambda_{n}$ is a Lagrange multiplier, and $v_{\Omega_{n}}(x)$ is the Riesz potential,

$$
v_{\Omega}(x):=\int_{\Omega} \frac{d y}{|x-y|^{s}} .
$$

In addition, by the proof of Theorem $2, \Omega_{n}$ is $C^{1, \alpha}$ close to the sets

$$
S_{n}:=\left[E^{0}+\bigcup_{i=1}^{N}\left(E^{i}+x_{n}^{i}\right)\right]
$$

in the sense that for all fixed $R>0$ with $E^{i} \subset \subset B_{R}(0)$,

$$
\partial^{*} \widetilde{\Omega}_{n}^{i}:=\left(\partial^{*} \Omega_{n}-x_{n}^{i}\right) \cap B_{R}(0) \rightarrow \partial^{*} E^{i} \quad \text { in } C^{1, \alpha},
$$

for all $\alpha \in\left(0, \frac{1}{2}\right)$, and the former are expressed as graphs over the limiting sets $E^{i}$,

$$
\partial^{*} \widetilde{\Omega}_{n}^{i}=\left\{y=\Psi_{n}(x):=x+\psi_{n}(x) \nu_{i}(x): x \in \partial^{*} E^{i}\right\},
$$

with $\psi_{n}(x) \rightarrow 0$ in $C^{1, \alpha}$ (see [1, Theorem 4.2].) As each $E^{i}$ is itself a minimizer of $\mathbf{E}_{0}$, by the above stated regularity theorem, $\partial^{*} E^{i}$ is of class $C^{3, \beta}$, and its normal vector $\nu_{E^{i}} \in C^{2}$. Finally, by [․ Proposition 2.1], the Riesz potentials $v_{\widetilde{\Omega}_{n}^{i}}$ are bounded in $C^{1, \beta}\left(B_{R}(0)\right)$, so along a subsequence they converge uniformly to $v_{E^{i}}$ in $B_{R}(0)$. 
For any $\zeta \in C_{0}^{\infty}\left(B_{R}(0) ; \mathbb{R}^{d}\right)$ we may integrate the Euler-Lagrange equations (40) by parts over $\partial^{*} \widetilde{\Omega}_{n}^{i}$,

$$
\int_{\partial^{*} \widetilde{\Omega}_{n}^{i}}\left(\operatorname{div}_{\tau_{n}} \zeta-\left(2 v_{\widetilde{\Omega}_{n}^{i}}-Z_{n}|x|^{-p}\right)\left(\zeta \cdot \nu_{n}\right)\right) d \mathcal{H}^{d-1}=\lambda_{n} \int_{\partial^{*} \widetilde{\Omega}_{n}^{i}} \zeta \cdot \nu_{n} d \mathcal{H}^{d-1}
$$

where $\nu_{n}:=\nu_{\widetilde{\Omega}_{n}^{i}}$ is the normal vector, and the tangential divergence on $\partial^{*} \widetilde{\Omega}_{n}^{i}$ is defined via

$$
\operatorname{div}_{\tau_{n}} \zeta=\operatorname{div} \zeta-\nu_{n} \cdot D \zeta \nu_{n}
$$

Using the parametrization $\Psi_{n}$ we can write the above equation with integrals over $\partial^{*} E^{i}$, with Jacobian $J_{n}=\left|\operatorname{det} D \Psi_{n}\right|$. As $\nu_{n} \rightarrow \nu_{E^{i}}$, we have $\operatorname{div}_{\tau_{n}} \zeta \rightarrow \operatorname{div}_{\tau_{E^{i}}} \zeta$, and $J_{n} \rightarrow 1$, by the $C^{1, \alpha}$ convergence and $\nu_{E^{i}} \in C^{2}$. Thus, we may pass to the limit $n \rightarrow \infty$ in the both integrals in (41) and obtain

$$
\begin{aligned}
\int_{\partial^{*} \widetilde{\Omega}_{n}^{i}}\left(\operatorname{div}_{\tau_{n}} \zeta-\left(2 v_{\widetilde{\Omega}_{n}^{i}}-Z_{n}|x|^{-p}\right)\left(\zeta \cdot \nu_{n}\right)\right) d \mathcal{H}^{d-1} \\
\longrightarrow \int_{\partial^{*} E^{i}}\left(\operatorname{div}_{\tau_{E^{i}}} \zeta-2 v_{E^{i}}\left(\zeta \cdot \nu_{E^{i}}\right)\right) d \mathcal{H}^{d-1}
\end{aligned}
$$

and

$$
\int_{\partial^{*} \widetilde{\Omega}_{n}^{i}} \zeta \cdot \nu_{n} d \mathcal{H}^{d-1} \longrightarrow \int_{\partial^{*} E^{i}} \zeta \cdot \nu_{E^{i}} d \mathcal{H}^{d-1}
$$

Thus, $\lambda_{n} \rightarrow \lambda_{0}$ for some limiting Lagrange multiplier $\lambda_{0}$. The values of $\lambda_{n}$ being (by (40) ) the same for each component of $\partial^{*} \Omega_{n}$, the value of $\lambda_{0}$ is independent of $i=0, \ldots, N$. Thus, the limiting curvature equation is the same for each limiting set $E^{i}$, notably with the same Lagrange multiplier $\lambda_{0}$. Since for $s<\bar{s}(d)$ the limiting sets $E^{i}$ are all balls (cf. [6. Theorem 2.11]), and the Lagrange multiplier is uniquely determined by the mass $m^{i}$ for balls, they must all have the same radius.

\section{REFERENCES}

[1] E. Acerbi, N. Fusco, and M. Morini, "Minimality via second variation for a nonlocal isoperimetric problem," Comm. Math. Phys., vol. 322, no. 2, pp. 515-557, 2013.

[2] S. Alama, L. Bronsard, R. Choksi, and I. Topaloglu, "Droplet phase in a nonlocal isoperimetric problem under confinement," arXiv preprint arXiv:1609.03589, 2016.

[3] — , "Ground-states for the liquid drop and TFDW models with long-range attraction," $J$. Math. Phys., vol. 58, no. 10, pp. 103 503, 11, 2017.

[4] A. Bahri, Critical points at infinity in some variational problems, ser. Pitman Research Notes in Mathematics Series. Longman Scientific \& Technical, Harlow; copublished in the United States with John Wiley \& Sons, Inc., New York, 1989, vol. 182.

[5] J. D. Bekenstein, "Relativistic gravitation theory for the modified Newtonian dynamics paradigm," Phys. Rev. D, vol. 70, p. 083509, 2004.

[6] M. Bonacini and R. Cristoferi, "Local and global minimality results for a nonlocal isoperimetric problem on $\mathbb{R}^{N}$," SIAM J. Math. Anal., vol. 46, no. 4, pp. 2310-2349, 2014.

[7] D. V. Bugg, "Mond-a review," Can. J. Phys., vol. 93, no. 2, pp. 119-125, 2015. 
[8] R. Choksi, C. B. Muratov, and I. Topaloglu, "An old problem resurfaces nonlocally: Gamow's liquid drops inspire today's research and applications," Notices Amer. Math. Soc., vol. 64, no. 11, pp. 1275-1283, 2017.

[9] R. Choksi and M. A. Peletier, "Small volume fraction limit of the diblock copolymer problem: I. Sharp-interface functional," SIAM J. Math. Anal., vol. 42, no. 3, pp. 1334-1370, 2010.

[10] — , "Small volume-fraction limit of the diblock copolymer problem: II. Diffuse-interface functional," SIAM J. Math. Anal., vol. 43, no. 2, pp. 739-763, 2011.

[11] L. Esposito and N. Fusco, "A remark on a free interface problem with volume constraint," $J$. Convex Anal., vol. 18, no. 2, pp. 417-426, 2011.

[12] A. Figalli, N. Fusco, F. Maggi, V. Millot, and M. Morini, "Isoperimetry and stability properties of balls with respect to nonlocal energies," Comm. Math. Phys., vol. 336, no. 1, pp. 441-507, 2015.

[13] A. Finzi, "On the validity of Newton's law at a long distance," Monthly Notices Roy. Astronom. Soc., vol. 127, pp. 21-30, 1963.

[14] R. L. Frank, R. Killip, and P. T. Nam, "Nonexistence of large nuclei in the liquid drop model," Lett. Math. Phys., vol. 106, no. 8, pp. 1033-1036, 2016.

[15] R. L. Frank and E. H. Lieb, "A compactness lemma and its application to the existence of minimizers for the liquid drop model," SIAM J. Math. Anal., vol. 47, no. 6, pp. 4436-4450, 2015.

[16] R. L. Frank, P. T. Nam, and H. van den Bosch, "The ionization conjecture in Thomas-FermiDirac-von Weizsäcker theory," Comm. Pure Appl. Math., to appear.

[17] G. Gamow, "Mass defect curve and nuclear constitution," Proc. R. Soc. Lond. A, vol. 126 , no. 803, pp. 632-644, 1930.

[18] H. Knüpfer and C. B. Muratov, "On an isoperimetric problem with a competing nonlocal term I: The planar case," Comm. Pure Appl. Math., vol. 66, no. 7, pp. 1129-1162, 2013.

[19] — "On an isoperimetric problem with a competing nonlocal term II: The general case," Comm. Pure Appl. Math., vol. 67, no. 12, pp. 1974-1994, 2014.

[20] H. Knüpfer, C. B. Muratov, and M. Novaga, "Low density phases in a uniformly charged liquid," Comm. Math. Phys., vol. 345, no. 1, pp. 141-183, 2016.

[21] E. H. Lieb and M. Loss, Analysis, ser. Graduate Studies in Mathematics. American Mathematical Society, Providence, RI, 1997, vol. 14.

[22] P.-L. Lions, "Solutions of Hartree-Fock equations for Coulomb systems," Comm. Math. Phys., vol. 109, no. 1, pp. 33-97, 1987.

[23] — , "The concentration-compactness principle in the calculus of variations. The locally compact case. I," Ann. Inst. H. Poincaré Anal. Non Linéaire, vol. 1, no. 2, pp. 109-145, 1984.

[24] J. Lu and F. Otto, "An isoperimetric problem with Coulomb repulsion and attraction to a background nucleus," arXiv preprint arXiv:1508.0717\%, 2015.

[25] — - "Nonexistence of a minimizer for Thomas-Fermi-Dirac-von Weizsäcker model," Comm. Pure Appl. Math., vol. 67, no. 10, pp. 1605-1617, 2014.

[26] F. Maggi, Sets of Finite Perimeter and Geometric Variational Problems, 1st ed., ser. Cambridge Studies in Advanced Mathematics. Cambridge: Cambridge University Press, 2012, vol. 135 .

[27] M. Milgrom, "A modification of the Newtonian dynamics as a possible alternative to the hidden mass hypothesis," The Astrophysical Journal, vol. 270, pp. 365-370, 1983.

[28] — " "Mond theory," Can. J. Phys., vol. 93, no. 2, pp. 107-118, 2015.

[29] X. Ren and J. Wei, "Double tori solution to an equation of mean curvature and Newtonian potential," Calc. Var. Partial Differential Equations, vol. 49, no. 3-4, pp. 987-1018, 2014.

[30] — - "The spectrum of the torus profile to a geometric variational problem with long range interaction," Phys. D, vol. 351/352, pp. 62-88, 2017. 
[31] M. Struwe, Variational methods, 4th ed., ser. Ergebnisse der Mathematik und ihrer Grenzgebiete. 3. Folge. A Series of Modern Surveys in Mathematics [Results in Mathematics and Related Areas. 3rd Series. A Series of Modern Surveys in Mathematics]. Springer-Verlag, Berlin, 2008, vol. 34, applications to nonlinear partial differential equations and Hamiltonian systems.

Department of Mathematics and Statistics, McMaster University, Hamilton, ON E-mail address: alama@mcmaster.ca

Department of Mathematics and Statistics, McMaster University, Hamilton, ON E-mail address: bronsard@mcmaster.ca

Department of Mathematics and Statistics, McGill University, Montréal, QC

E-mail address: rustum.choksi@mcgill.ca

Department of Mathematics and Applied Mathematics, Virginia Commonwealth UNIVERSITY, RICHMOND, VA

E-mail address: iatopaloglu@vcu.edu 